\title{
Electromagnetic Design of Broadband Antenna Feed Systems for the Northern Cross Radio Telescope
}

\author{
G. Virone ${ }^{1}$, G. Addamo ${ }^{1}$, R. Tascone ${ }^{1}$, O. A. Peverini ${ }^{1}$, F. Perini ${ }^{2}$, M. Schiaffino ${ }^{2}$, G. Naldi ${ }^{2}$, J. Monari ${ }^{2}$, and \\ S. Montebugnoli ${ }^{2}$ \\ 1 IEIIT-CNR, c/o Politecnico di Torino, C.so Duca degli Abruzzi 24, 10129, Turin, Italy, http://www.to.ieiit.cnr.it \\ e-mail: giuseppe.virone@polito.it, giuseppe.addamo@polito.it, riccardo.tascone@polito.it, oscar.peverini@polito.it \\ 2 IRA-INAF, Via Fiorentina n. 3508/B - 40059 Fiorentina di Medicina, Bologna, Italy, http://www.med.ira. \\ inaf.it/index_EN.htm e-mail: f.perini@ira.inaf.it, mschiaffino@ira.inaf.it, gnaldi@ira.inaf.it, j.monari@ira.inaf.it, \\ s.montebugnoli@ira.inaf.it *
}

\begin{abstract}
This contribution deals with the design and implementation of broadband antenna feed systems for the cylindrical offset parabolic reflector of the Northern Cross radio telescope, in the framework of the Square Kilometer Array Design Study (SKADS-FP6) project. Such feed systems have to maintain a proper radiation pattern as well as a good matching condition in the operative frequency band $(120-430 \mathrm{MHz})$ in order to efficiently feed the main reflector. This task is not trivial since more than octave bandwidths are required. Several feed system designs have been carried out on the basis of different architectures such as log periodic antenna arrays, linear arrays of fat dipoles and branched Vivaldi (tapered slot) radiators inside a wired subreflector. Each configuration provides a different trade-off between electrical performance and manufacturing complexity. The most significant computed and experimental results are discussed. Some of the proposed configurations have already been mounted on the Northern Cross Radio Telescope. The adopted mechanical and electronic solutions such as wiring, canalization, amplifier housing and protection are reported. A preliminary study on the application of Focal Plane Arrays on the E/W Arm is also discussed.
\end{abstract}

\section{Introduction}

The Italian contribution to the SKADS-FP6 project is focused on the development of both high-performance and low-cost antenna and receiver systems. In this framework, the existing Northern Cross Radio Telescope is being used as the main test bed (Bolli et al. 2008). This structure can in fact allow experiments with a very large collecting area (up to $30000 \mathrm{~m}^{2}$ ).

The Northern Cross Radio Telescope (see Fig. 1) is composed of a large cylindrical offset parabolic reflector called East/West Arm and a linear array of smaller cylindrical parabolic reflectors, called North/South Arm.

The cylindrical reflector illumination is always performed by means of a linear array of radiators placed along the focal axis. These radiators have to exhibit a low reflection coefficient and a suitable radiation pattern to feed the reflector with high efficiency in the operative frequency band. This task is not trivial when low operative frequencies and very large frequency bands are needed.

Furthermore, the mounting of both the antenna and receiver arrays on the actual reflector is a very important issue when efficient connection, mechanical stability in various weather condition and low-cost requirements are addressed.

\footnotetext{
^ This work was supported by the European Commission Framework Program 6, Project SKADS, Square Kilometre Array Design Studies (SKADS), contract no 011938.
}

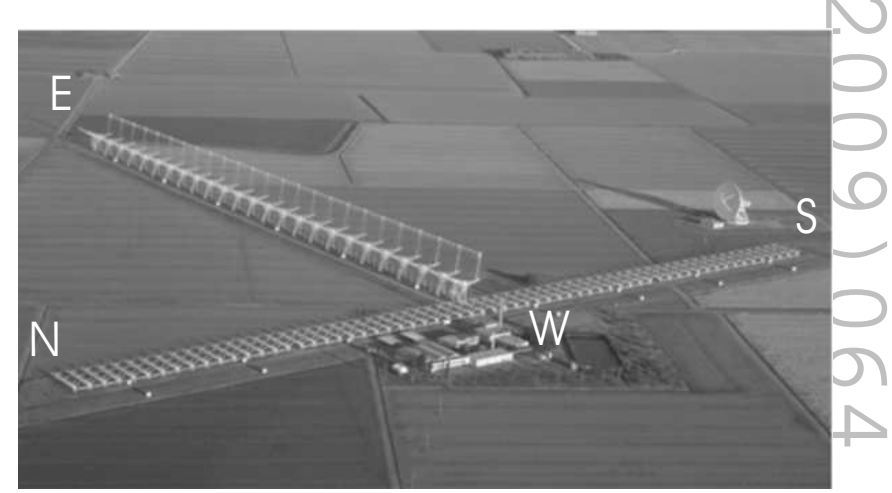

Fig. 1: North/South and East/West Arms of the Northern Cross Radio Telescope.

\section{Designed Broad Band Antenna Feed Systems}

The design work on the feed system for the Northern Cross Radio Telescope started with a careful electromagnetic analysis of both the North/South and East/West Arms by means of a proprietary simulation tool. This tool has been developed on the basis of the Pocklington's equation. The considered feed systems working at $408 \mathrm{MHz}$ were composed of a linear dipole arrays inside wired subreflectors. Specifically, a corner-style subreflector was featured on the E/W Arm offset reflector.

Subsequently, a similar architecture has been designed in the $120-240 \mathrm{MHz}$ band providing good results in terms of both reflection coefficient and radiation pattern. Specifically, larger fat dipoles and a bigger corner reflector were adopted to ob- 
tain such a low operative frequency and the octave bandwidth. However, this structure does not guarantee the compatibility with the present operative frequency $408 \mathrm{MHz}$. Moreover, the dipole inter-element spacing, which is about half wavelength at $120 \mathrm{MHz}$, does not provide a grating lobe free condition when the beam is scanned from broadside (in the E-plane) in the overall $120-240 \mathrm{MHz}$ band.

In order to overcome these problems, a new antenna system operating in a larger frequency band $120-430 \mathrm{MHz}$ has been developed. The system scans in angular range from $-30^{\circ}$ to $30^{\circ}$ in absence of grating lobes up to $240 \mathrm{MHz}$. This antenna system consists of a linear array of Vivaldi antennas (Langley et al. 1996; Shin \& Schaubert 1999) placed in a wired corner reflector. Each radiator is connected to the adjacent radiators and to the back of the subreflector. A slot line stub provides a symmetrical excitation of the tapered slot. The design of this system has been carried out by a modal approach similar to the design of a ridged horn antenna. The actual array presents an active reflection coefficient for uniform excitation lower than $-15 \mathrm{~dB}$ from 120 to $430 \mathrm{MHz}$.

The radiation patterns in the $H$-plane of the Vivaldi array (primary radiation pattern) and the overall optical system (secondary radiation pattern) have been computed at the frequencies $120 \mathrm{MHz}, 240 \mathrm{MHz}$ and $408 \mathrm{MHz}$. At $120 \mathrm{MHz}$, the tapering on the offset reflector due to the illumination is only $6 \mathrm{~dB}$. It is not possible to increase the tapering level due to the mechanical constrains on the dimensions and weight of the subreflector. Hence, the secondary pattern exhibits significant spillover lobes $(-17 \mathrm{~dB})$. The spillover lobes are below $-26 \mathrm{~dB}$ at the higher frequencies. The Full Half Power Beamwidth of the secondary pattern is $5^{\circ}$ at $120 \mathrm{MHz}, 2.5^{\circ}$ at $240 \mathrm{MHz}$ and $1.5^{\circ}$ at $408 \mathrm{MHz}$.

A subreflectorless structure is however cheaper and easier to integrate on the present radio telescope. Therefore, a custom $\log$ periodic antenna has been designed in the $120-240 \mathrm{MHz}$ frequency band, with a higher directivity with respect to the commercial ones, in order to obtain a reasonable illumination efficiency on the main reflector. The designed structure has 18 elements and is 2.2 meters long. The weight is $3.7 \mathrm{Kg}$.

An array of 18 radiators has been manufactured and installed on the E/W Arm of the Northern Cross Radio Telescope (see section 3). The simulated and measured reflection coefficients reported in Fig. 2 show a good 50- $\Omega$ impedance matching in a frequency band that is slightly larger than 120$240 \mathrm{MHz}$. A good agreement has also been obtained between the measured and simulated radiation patterns.

\section{Mechanical and Electrical Implementation}

The East/West Arm feed system has been modified to operate in the $120-240 \mathrm{MHz}$ frequency range. The modification consisted in removing the entire former focal line from sector 3 of the channel 1E. The line was replaced with an array of $18 \log$ periodic antennas connected via coaxial cables to waterproof boxes (see Fig. 3). As shown in Fig. 4, each box contains two frontends, two analog optical transmitters, one micro-controller and one power supply so it receives the signals coming from two antennas. A canalization structure was built to bring optical

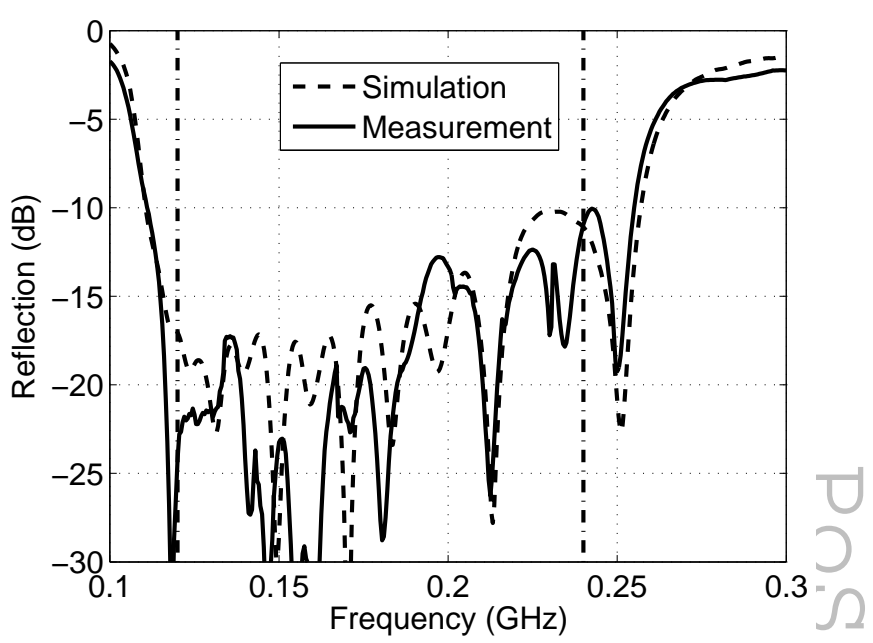

Fig. 2: Computed and measured reflection coefficient of the manufactured $\log$-periodic antenna prototypes.

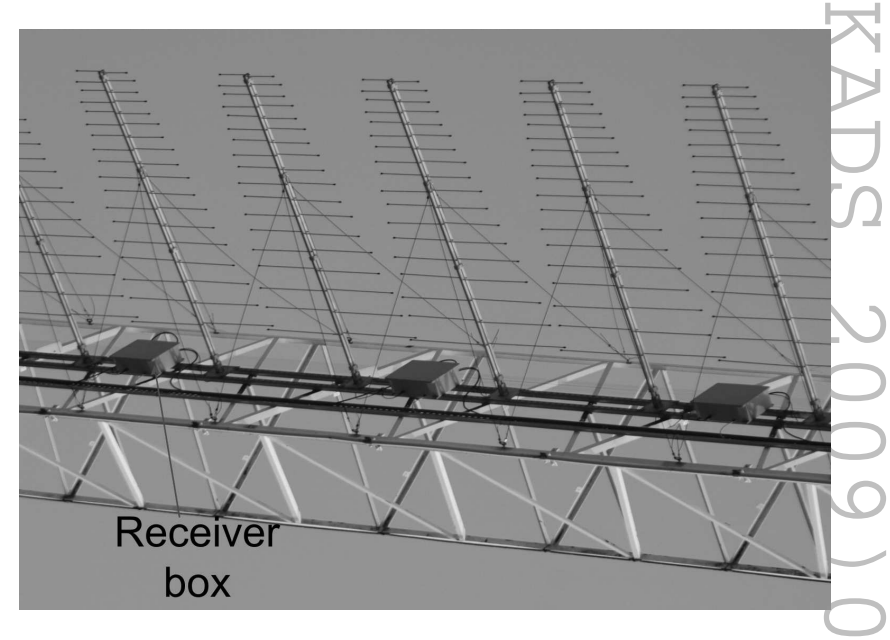

Fig. 3: Log periodic antenna array connected via coaxial cables to waterproof boxes.

fibers and power supply wiring to the data acquisition system in a safe manner.

\section{Focal Plane Array preliminary study}

The effects of a Focal Plane Array illumination on the E/W arm of the Northern Cross has also been preliminarily investigated. Specifically, the E/W main reflector has been illuminated with different field distributions in order to scan the beam in the $H$ plane with good overall antenna efficiency. The considered field distributions, which can be provided by an antenna array with the proper beam forming/combining network, simulate an aperture antenna whose phase center is placed in various positions within the focus area. This operation yields two contour plots showing the obtained beam deviation in the $H$-plane (Fig. 5) and the corresponding antenna efficiency (Fig. 6). As expected, the beam of the overall antenna can be scanned in the $H$-plane at the expense of the antenna efficiency. However, it can be ob- 


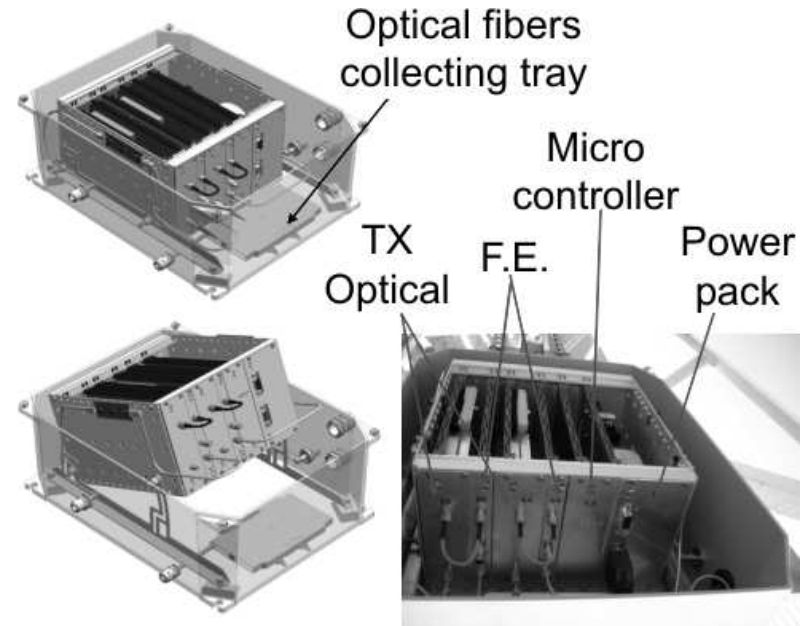

Fig. 4: Waterproof box containing two front-ends, two analog optical transmitters, one micro-controller and one power supply.

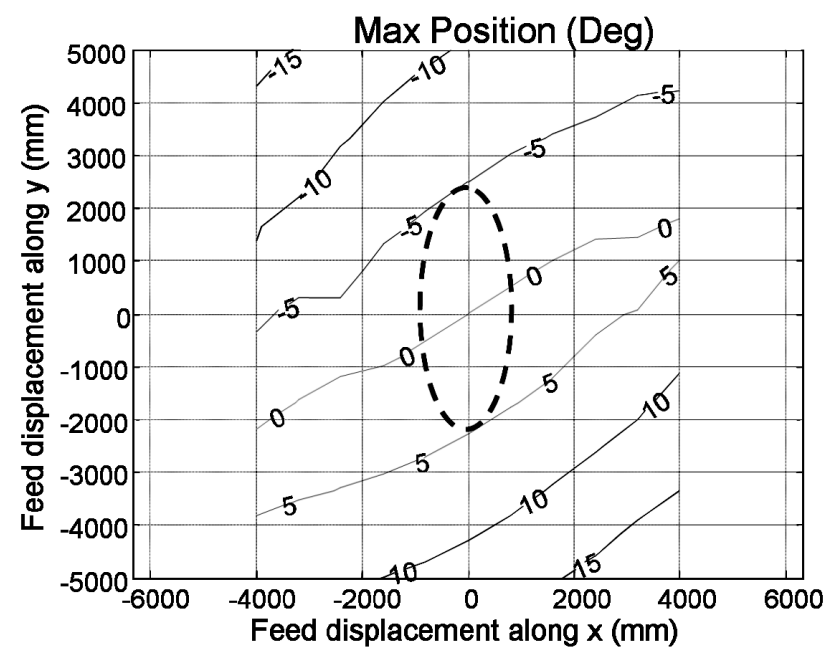

Fig. 5: Iso-level curves of the maximum radiation pattern angular position.

served that $\mathrm{a} \pm 5^{\circ}$ scanning angle is possible with a negligible efficiency loss.

\section{Conclusions}

The update of the Northern Cross Radio Telescope to wideband multi-receiver technologies produced several interesting advancements in the antenna and receiver design and manufacturing skills. Nevertheless, the developed systems can still be improved in terms of both high performance and reduced manufacturing complexity. Specifically, a novel mechanical implementation of the Vivaldi architecture is presently under investigation.

\section{References}

P. Bolli, F. Perini, S. Montebugnoli, G. Pelosi, S. Poppi, "Basic Element for Square Kilometer Array Training (BEST):

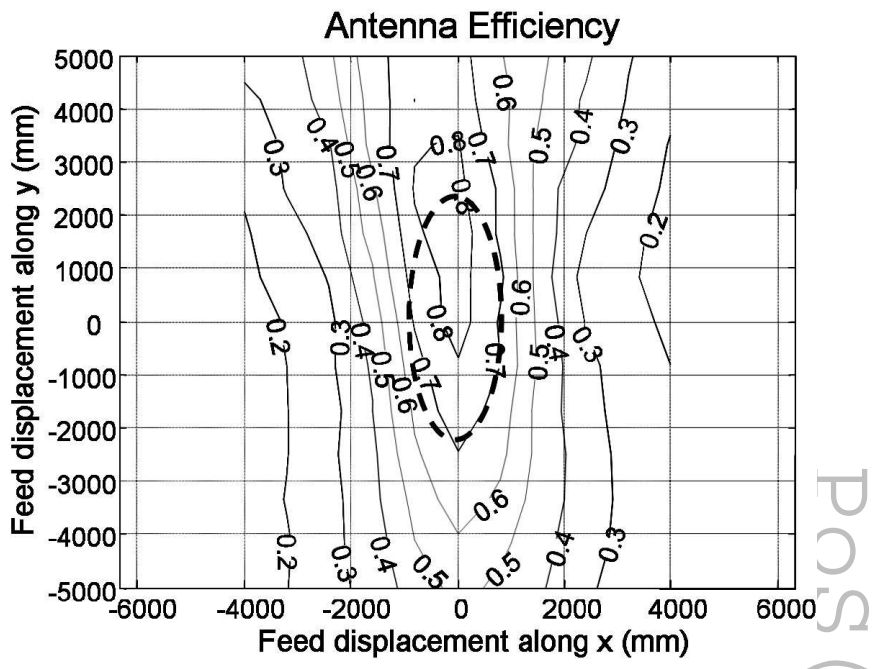

Fig. 6: Iso-level curves of the computed antenna efficiency.

Evaluation of the Antenna Noise Temperature," Antennas and Propagation Magazine, IEEE Volume 50, Issue 2, April 2008, Page(s): 58 - 65

J. Langley, P. S. Hall and P. Newham, "Balanced Antipodal Vivaldi Antenna for Wide Bandwidth Phased Arrays," IEE Proc. Microwaves, Antennas and Propagation, Vol 143, No. 2, April 1996.

J. Shin and D. H. Schaubert, "A Parameter Study of StriplineFed Vivaldi Notch Antenna Arrays," IEEE Transactions on Antenna and Propagation, Vol. 47, No. 5, May 1999, pp. 879-886 